\title{
High Grade Transformation in Adenoid Cystic Carcinoma of the Parotid: Report of a Case with Cytologic, Histologic and Immunohistochemical Study
}

\author{
Kiran Preet Malhotra • Vinita Agrawal • \\ Rakesh Pandey
}

Received: 16 April 2009/Accepted: 29 May 2009/Published online: 20 June 2009

(c) Humana 2009

\begin{abstract}
Adenoid cystic carcinoma (ACC) constitutes about $4 \%$ of salivary epithelial tumors and is the second common malignant epithelial salivary gland tumor involving both the major and minor salivary glands. High grade transformation in ACC is a recently recognized entity with only a few cases reported in literature. We report the first case of ACC with high grade transformation involving the parotid. A 54-year-old man with a history of right parotid painful swelling from 1.5 years presented with recent increase in size of the swelling and facial paresis of 2 months duration. The locally invasive salivary neoplasm was composed predominantly of an undifferentiated carcinoma with foci of conventional ACC occupying less than $10 \%$ of tumor area. Immunohistochemical study of the undifferentiated component as compared to conventional ACC showed greater over-expression of p53 and Ki-67. Her-2/Neu was negative in both components. Recognition of occasional clusters of basaloid cells and hyaline globules in association with larger poorly differentiated malignant cell population in aspiration smears can help in cytological diagnosis. The acquisition of high proliferation index and over-expression of p53 may be the probable factors involved in the pathogenesis of high grade transformation in a conventional ACC.
\end{abstract}

Keywords Carcinoma - Adenoid cystic · Parotid . Pathology $\cdot$ Cytology $\cdot$ p53 $\cdot$ Ki-67

K. P. Malhotra · V. Agrawal $(\bowtie) \cdot$ R. Pandey Department of Pathology, Sanjay Gandhi Postgraduate Institute of Medical Sciences, Raebareli Road, Lucknow, Uttar Pradesh 226014, India

e-mail: vinita@sgpgi.ac.in; vinita_pgi@yahoo.com

\section{Introduction}

Adenoid cystic carcinoma (ACC) represents the second most common malignancy of the salivary glands and is associated with a poor long term prognosis. It is composed of basaloid cells with primarily myoepithelial/basal cell differentiation. Cribriform, tubular and solid are the three recognized morphologic patterns. One of the important prognostic factors is the histological grade determined by the percentage of solid component in the tumor [1-3]. High grade transformation in ACC is a recently defined entity, which is characterized by areas of conventional adenoid cystic carcinoma associated with concomitant presence of undifferentiated or poorly differentiated carcinoma [4]. However, this has not yet been included in the grading schemes for salivary ACC. Only a few cases have been reported in the literature involving minor salivary glands and submandibular gland [4-10]. We report the first case of ACC with high grade transformation arising in the parotid. Helpful cytologic clues, histological features and immunohistochemical findings are discussed.

\section{Clinical History}

A 54-year-old male presented at a tertiary care center of North India with complaints of a painful mass in the right infra-auricular region for 1.5 years. The patient had noted a recent increase in the size of the mass and associated facial paresis for the last 2 months. On examination, he had a hard mass in the right parotid with restricted mobility and facial nerve involvement. A computed tomogram showed an enhancing mass lesion with infiltration of adjoining soft tissue. The mandible was uninvolved. Fine needle aspiration cytology was performed, followed by surgical 
resection of the tumor, histological examination and immunohistochemical work-up.

\section{Materials and Methods}

\section{Cytology}

Fine needle aspiration was performed using 22 gauge needles from multiple sites. No separate lymph node was identified. The smears were stained with May Grunwald Giemsa, Hematoxylin and Eosin and Papanicolaou stains.

\section{Histology}

A parotidectomy specimen was received. Multiple sections were taken from various areas of the tumor. Adequate representation of all areas with varied gross morphology was ensured. Formalin-fixed paraffin-embedded tissue was sectioned at 2-4 $\mu \mathrm{m}$ and stained with Hematoxylin and Eosin. The conventional adenoid cystic areas were graded according to the three tier grading system of Szanto et al. [3].

\section{Immunohistochemistry}

Immunohistochemistry was performed on formalin-fixed paraffin-embedded tissue. Primary antibodies used (Dako, Denmark) included cytokeratin (CK) and epithelial membrane antigen (EMA) as markers of epithelial cell differentiation and S-100 and smooth muscle actin (SMA) as markers of myoepithelial cell differentiation. The markers to analyze the biological behavior of the tumor included CD117, a c-kit proto-oncogene product, E-Cadherin, a cell adhesion protein, Her-2/Neu, a transmembrane tyrosine kinase receptor and p53 protein, a tumor suppressor gene product. The cell proliferation marker, Ki-67 was also studied. p53 and Ki-67 were assessed quantitatively in both the conventional ACC and high-grade areas. The reaction was visualized using the sensitive enzyme conjugated polymer-based detection system (Envision visualization system, Dako, Denmark). DAB (diaminobenzidine) was used as chromogen (Dako, Denmark). Adequate positive and negative controls, which stained appropriately, were also simultaneously stained.

\section{Results}

The fine needle aspiration smears were cellular with dispersed and few non-cohesive clusters of atypical cells on a hemorrhagic background. The atypical cells had intermediate to large, round to pleomorphic hyperchromatic nuclei, coarse chromatin, conspicuous nucleoli and variable amount of poorly defined cytoplasm. Occasional hyaline stromal globules were also identified (Fig. 1). Differential diagnoses of adenocarcinoma (not otherwise specified), undifferentiated carcinoma, salivary duct carcinoma, basaloid adenocarcinoma and carcinoma ex pleomorphic adenoma were entertained.

On surgical resection, the tumor was vascular, encasing the facial nerve and infiltrating the subcutaneous tissue and adjoining fat planes. On gross examination, the tumor measured $5 \times 4 \times 2 \mathrm{~cm}$ and replaced the entire parotid except for a thin wedge of grossly uninvolved tissue at one side. The cut surface of the tumor was variegated with solid, cystic, hemorrhagic and necrotic areas. On microscopic examination the tumor was primarily composed of sheets and nests of cells with intermediate to large-sized hyperchromatic nuclei, prominent nucleoli, scant eosinophilic cytoplasm and frequent mitosis (>20/10 hpf) (Fig. 2). The tumor nests were devoid of surrounding myoepithelial cells. Areas of necrosis $(\sim 10 \%$ of tumor area) and desmoplasia were seen. No intratumoral calcification was present. A discrete focus of conventional adenoid cystic carcinoma (grade II) occupying $<10 \%$ area of the studied sections was identified. The tumor cells in this focus were arranged entirely in cribriform pattern showing small basaloid cells with interspersed pseudocystic spaces containing fuschinophilic acellular hyaline material and infrequent mitosis ( $\sim 1 / 10 \mathrm{hpf}$; Fig. 3). Extensive perineural and lymphovascular invasion by tumor cells was seen. The tumor was diffusely infiltrating the adjoining salivary gland, fibrofatty tissue and skeletal muscle. A single lymph node was identified in the attached fat, metastatic deposits in which showed presence of high grade areas along with few foci of conventional ACC. A

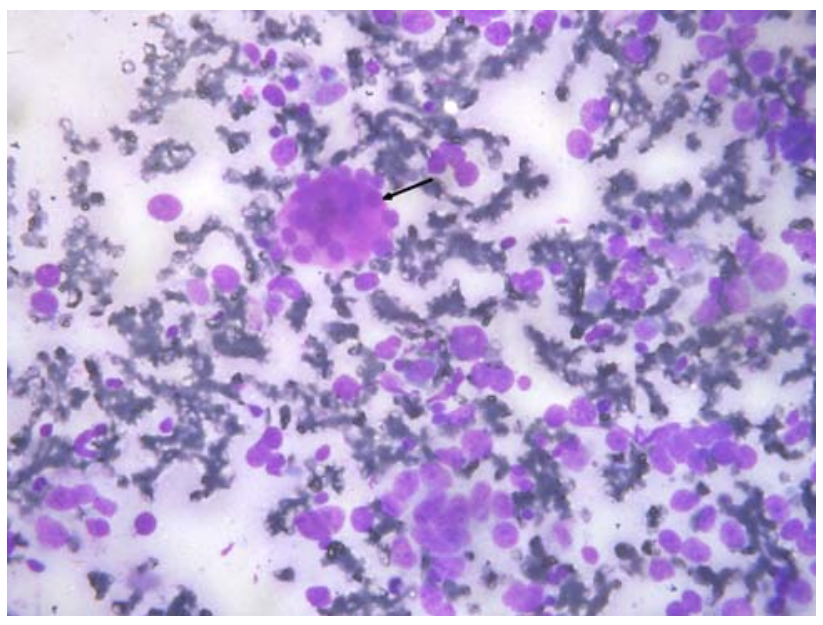

Fig. 1 FNA smears showing dispersed population of highly pleomorphic cells with a small cluster of isomorphic basaloid cells surrounding a hyaline globule (arrow) (MGG) 


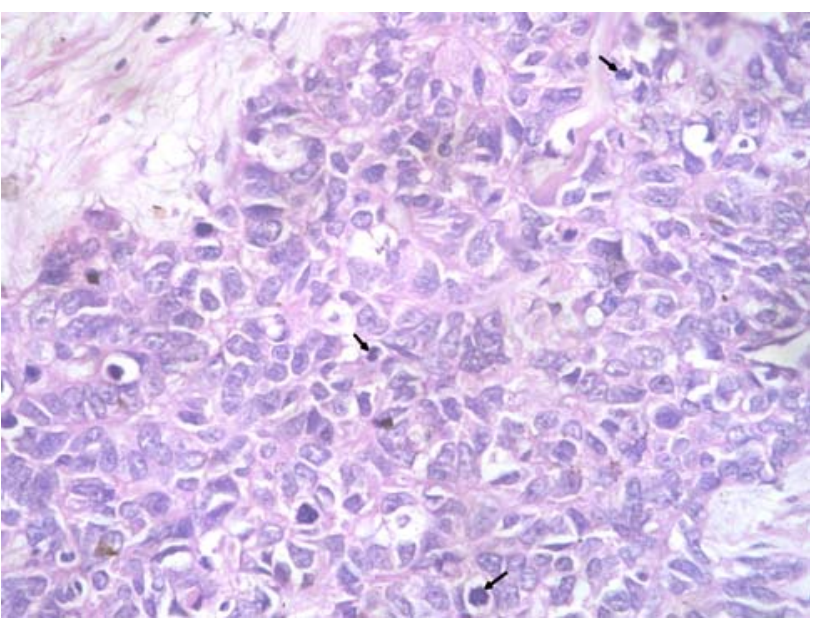

Fig. 2 High grade ACC component showing pleomorphic undifferentiated cells with frequent mitoses (arrows)

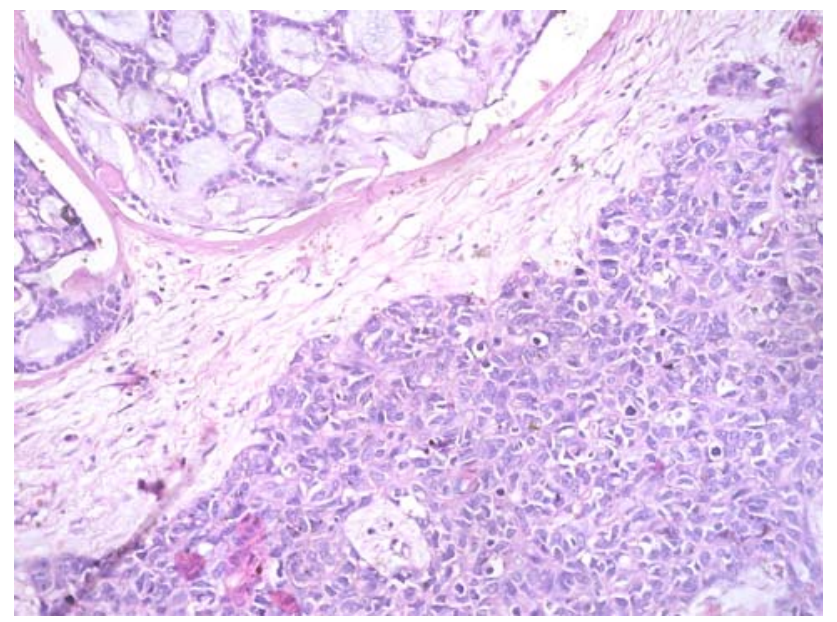

Fig. 3 Conventional ACC component with isomorphic basaloid cells in cribriform pattern (upper left) and high-grade undifferentiated ACC component (lower right)

retrospective review of the fine needle aspirate smears revealed few small aggregates of isomorphic basaloid cells.

On immunohistochemistry the tumor was diffusely positive for cytokeratin, epithelial membrane antigen and S-100 in both the components. Smooth muscle actin was confined to the abluminal cells in the differentiated component but was negative in the high-grade component. This was interpreted as presence of both epithelial and myoepithelial differentiation in the conventional ACC areas, while there was loss of myoepithelial differentiation in the high grade areas. p53 expression was seen in $20 \%$ tumor cells of conventional ACC and was of moderate intensity. However the high-grade component displayed strong nuclear p53 positivity in $\sim 60 \%$ of tumor cells. Ki-67 staining showed a cell proliferation index of $<10 \%$ in conventional ACC and 50\% in the high-grade areas

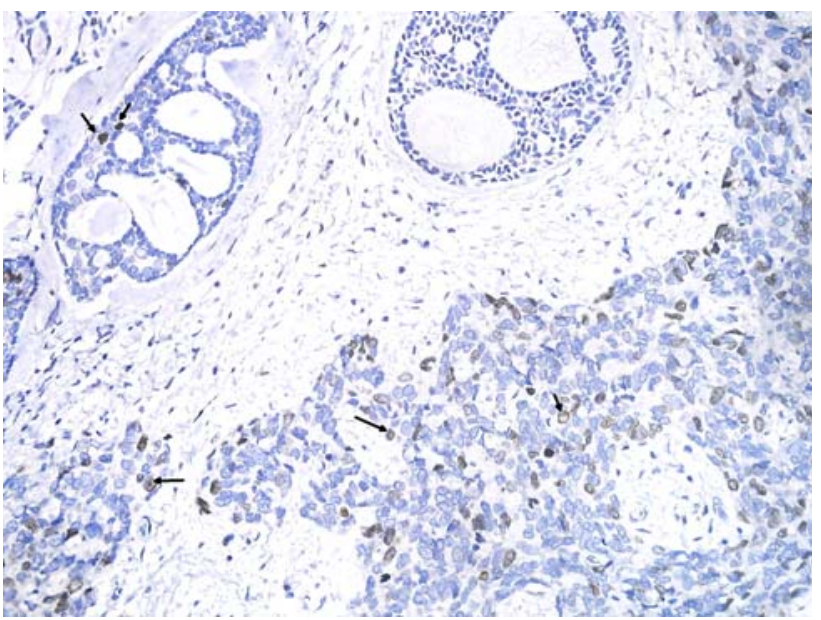

Fig. 4 Nuclear positivity for Ki67 (arrows) in greater proportion of cells in high-grade component (lower right) as compared to conventional component (upper left) (DAB)

Table 1 Comparison of histology and immunohistochemical features in differentiated and high-grade components

\begin{tabular}{|c|c|c|}
\hline & $\begin{array}{l}\text { Differentiated } \\
\text { component }\end{array}$ & $\begin{array}{l}\text { High grade } \\
\text { component }\end{array}$ \\
\hline Relative percentage & 10 & 90 \\
\hline Histology & $\begin{array}{l}\text { Conventional ACC } \\
\text { grade II }\end{array}$ & $\begin{array}{l}\text { Undifferentiated } \\
\text { carcinoma }\end{array}$ \\
\hline Relative nuclear size & 1 & $2-4$ \\
\hline Mitosis/10 hpf & 1 & $>20$ \\
\hline CK, EMA, S-100 & + & + \\
\hline SMA & $+($ abluminal cells $)$ & - \\
\hline p53 intensity & $\begin{array}{l}\text { Moderate in } 20 \% \\
\text { cells }\end{array}$ & Strong in $60 \%$ cells \\
\hline Ki67 index & $<10 \%$ & $50 \%$ \\
\hline Her-2/Neu & - & - \\
\hline E Cadherin & + & + \\
\hline C Kit & + & + \\
\hline
\end{tabular}

(Fig. 4). E-Cadherin and c-kit (CD117) were positive in both the conventional and high-grade areas. Both components had no Her-2/Neu expression.

A diagnosis of adenoid cystic carcinoma with high grade transformation was made. Table 1 shows the comparative features of both components. The patient was given postoperative local radiotherapy. Five months of follow-up since surgery showed no recurrence or evidence of distant metastasis.

\section{Discussion}

Adenoid cystic carcinoma (ACC) is an uncommon form of malignant neoplasm that arises most commonly in the 
major and minor salivary glands of the head and neck. It is often slow to metastasize, but has a persistent and relentless growth with a poor long term prognosis. Major and minor salivary glands including seromucinous glands of upper respiratory tract are the frequent sites of occurrence. The histological picture is variable. In its classic form, the tumor is composed of small uniform cells arranged in cribriform, tubular or solid growth patterns with interspersed globules and cylinders of hyaline basement membrane material [1].

Szanto et al. [3] have defined a three tier grading system which takes into account the histomorphologic pattern and percentage of the solid component. This has been regarded as a useful prognostic indicator [2, 3]. Cheuk et al. [5] first used the term 'dedifferentiated adenoid cystic carcinoma' to describe areas with pleomorphic tumor foci in close proximity to areas of conventional adenoid cystic carcinoma. Seethala et al. [4] have reported eleven new cases with similar morphology and are proponents of the term 'adenoid cystic carcinoma with high grade transformation'. This encompasses tumors showing a high grade carcinoma with either a conventional low grade ACC as described by Cheuk, or a solid variant of conventional ACC as described by Sato et al. as the associated component $[5,10]$.

Pubmed search revealed a total of 24 cases of dedifferentiated ACC and ACC with high grade transformation. Of these, 17 occurred in minor salivary glands of tongue, palate and sinonasal tract including a tumor in the hypopharynx $[5,6,8-10]$. Seven tumors occurred in the submandibular gland [7, 9, 10]. We report the first case from parotid. The parotid gland, though the commonest site of conventional ACC has not been reported earlier as a site of high grade transformation of ACC.

Our case showed morphology similar to the 'dedifferentiated ACC' described by Cheuk et al. [5]. The transformed component showed prominent anisonucleosis, conforming to the criteria established by Seethala et al. [4], requiring more than twofold nuclear enlargements as compared to the areas of conventional ACC. Micropapillae and squamoid areas have been reported, but were not seen in our case $[4,5]$.

The cytological features of ACC with high-grade transformation have not been reported earlier in the literature. We considered the cytological differential diagnosis of adenocarcinoma (not otherwise specified), undifferentiated carcinoma, salivary duct carcinoma, basaloid adenocarcinoma and carcinoma ex pleomorphic adenoma based on presence of a population of poorly differentiated atypical cells with occasional hyaline globules. On review, the aspirate smears were found to have small groups of basaloid cells, presumably derived from the conventional ACC component. It needs to be emphasized that a thorough analysis of fine needle aspirate smears in such cases may reveal groups of basaloid cells and hyaline globules amidst a highly pleomorphic population which would be pointers to a diagnosis of ACC with high-grade transformation. Preoperative aspiration diagnosis in such cases would be of value in deciding the extent of surgery and also promote thorough sectioning and sampling of tumor tissue received, since conventional ACC areas may represent only a minor component. In our case, the conventional ACC areas comprised a total of $10 \%$ of tumor tissue. Other studies have reported similar figures [4, 5, 7, 9].

Immunohistochemical analysis revealed loss of myoepithelial markers in the high-grade component as reported in earlier studies [4, 5, 9]. The transformed component showed a Ki-67 labeling index of $50 \%$ and p53 overexpression in $60 \%$ of tumor cells which was significantly greater than that in the conventional ACC component. Similar findings have been reported earlier and attest to the morphologic picture as well as clinical outcome of such cases [5, 7-10]. C-kit was positive in both conventional and high-grade areas and did not help in discriminating between the two components as described in previous reports $[4,5,7,9]$. E-Cadherin expression, which has not been reported earlier, was seen in both the components.

We found negative Her-2/Neu in both components. Previous studies have reported variable results of Her-2/ Neu in the high grade component [4, 9, 10]. However, conventional ACC is uniformly negative. Her-2/Neu negativity has been surmised as a helpful feature in ruling out the close differential of a hybrid ACC-salivary duct carcinoma [4].

In conclusion, we present the first report of adenoid cystic carcinoma with high-grade transformation arising in the parotid gland. The cytologic findings, as yet undescribed, are delineated. We emphasize that thorough study of aspirate smears may reveal clusters of basaloid cells and hyaline globules in a highly pleomorphic tumor cell population that may help to clinch a difficult differential diagnosis and guide extent of surgery and tissue sampling post-surgery. Higher p53 and Ki67 expression in the highgrade component with associated loss of myoepithelial markers, suggest their possible role in pathogenesis.

\section{References}

1. Barnes EL, Eveson JW, Reichart P, Sidransky D, editors. Pathology and genetics of head and neck tumours. Kleihues P, Sobin LH, series editors. World Health Organization Classification of Tumours. Lyon, France: IARC Press; 2005.

2. da Cruz Perez DE, de Abreu Alves F, Nobuko Nishimoto I, et al. Prognostic factors in head and neck adenoid cystic carcinoma. Oral Oncol. 2005;42:139-46. doi:10.1016/j.oraloncology.2005. 06.024 . 
3. Szanto PA, Luna MA, Tortoledo ME, et al. Histologic grading of adenoid cystic carcinoma of the salivary glands. Cancer. 1984;54:1062-9. doi:10.1002/1097-0142(19840915)54:6<1062:: AID-CNCR2820540622>3.0.CO;2-E.

4. Seethala RR, Hunt JL, Baloch ZW, et al. Adenoid cystic carcinoma with high-grade transformation a report of 11 cases and a review of the literature. Am J Surg Pathol. 2007;31:1683-94. doi: 10.1097/PAS.0b013e3180dc928c.

5. Cheuk W, Chan JK, Ngan RK. Dedifferentiation in adenoid cystic carcinoma of salivary gland: an uncommon complication associated with an accelerated clinical course. Am J Surg Pathol. 1999;23:465-72. doi:10.1097/00000478-199904000-00012.

6. Moles MA, Avila IR, Archilla AR. Dedifferentiation occurring in adenoid cystic carcinoma of the tongue. Oral Surg Oral Med Oral Pathol Oral Radiol Endod. 1999;88:177-80. doi:10.1016/S10792104(99)70114-9.
7. Chau Y, Hongyo T, Aozasa K, et al. Dedifferentiation of adenoid cystic carcinoma: report of a case implicating p53 gene mutation. Hum Pathol. 2001;32:1403-7. doi:10.1053/hupa.2001.28966.

8. Ide F, Mishima K, Saito I. Small foci of high-grade carcinoma cells in adenoid cystic carcinoma represent an incipient phase of dedifferentiation. Histopathology. 2003;43:604-6. doi:10.1111/ j.1365-2559.2003.01682.x.

9. Nagao T, Gaffey TA, Serizawa H, et al. Dedifferentiated adenoid cystic carcinoma: a clinicopathologic study of 6 cases. Mod Pathol. 2003;16:1265-72. doi:10.1097/01.MP.0000097366.88165.08.

10. Sato K, Ueda Y, Sakurai A, et al. Adenoid cystic carcinoma of the maxillary sinus with gradual histologic transformation to highgrade adenocarcinoma: a comparative report with dedifferentiated carcinoma. Virchows Arch. 2006;448:204-8. doi:10.1007/ s00428-005-0054-8. 